\title{
The LAGO (Large Aperture GRB Observatory) in Peru
}

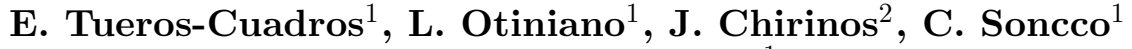 \\ and W. Guevara-Day ${ }^{1}$ \\ ${ }^{1}$ Departamento de Astrofísica, Agencia Espacial del Perú, CONIDA \\ ${ }^{2}$ Michigan Technology University
}

\begin{abstract}
The Large Aperture GRBs Observatory is a continental-wide observatory devised to detect high energy (around $100 \mathrm{GeV}$ ) component of Gamma Ray Bursts (GRBs), by using the single particle technique in arrays of Water Cherenkov Detectors (WCDs) at high mountain sites of Argentina, Bolivia, Colombia, Guatemala, Mexico, Venezuela and Peru. Details of the instalation and operation of the detectors in Marcapomacocha in Peru at 4550 m.a.s.l. are given. The detector calibration method will also be shown.
\end{abstract}

Keywords. GRB, Gamma Rays.

\section{Introduction}

The LAGO (Large Aperture GRBs Observatory) international collaboration goal is to observe high energy component (around $100 \mathrm{GeV}$ ) of Gamma Ray Bursts (GRBs) through Water Cherenkov Detectors (WCDs) in high mountain sites, (Bertou 2008) using the single particle technique (Aglietta et al. 1996). When a GRB occurs, the atmosphere is impacted by a multitude of high energy gamma rays that produce decay showers. Together those showers are detectable at ground level as an instant excess in the flux measured by a detector. Gamma ray showers have a composition of $90 \%$ photons, $9 \%$ electrons and $<1 \%$ muons, WCDs are sensitive to all those particles. These showers are absorbed high in the atmosphere, not being able to survive and reach the ground. In order to detect the greatest possible number of particles, the water Cherenkov tank must be placed at high altitude. Peru is in an initial phase. We have built two detectors, located at 4450 m.a.s.l. in Marcapomacocha-Huancayo, and a third one is under construction in the same location.

\section{Experimental Setup}

The LAGO project is using electronic acquisition and photomultiplier tubes (PMTs) from the engineering array of the Pierre Auger Observatory (PAO), (Pierre Auger Collaboration 2004). As data storage, we use an ARM computer which has a low power consumption (less than 1 watt) and resists extreme temperatures $\left(-40^{\circ} \mathrm{C}\right.$ to $\left.85^{\circ} \mathrm{C}\right)$. At high altitude locations in Peru it is dificult to get stable power sources but the low consumption requirements of this system allows the use of solar panels and batteries. The WCD consists of a commercial cylindrical poliethylene tanks of $4 m^{2}$, with a large area PMT located in the central upper section. It has an internal cover of banner to ensure a good reflexivity and diffusivity and it is shielded from external light. The WCDs are filled with clear water up to $1.3 \mathrm{~m}$ in order to ensure a high probability of photon conversion inside the tank. The water treatment must be done on the site. Filtered water is collected in a poliethylene tank and treated with aluminum sulfate, then is left to sediment 

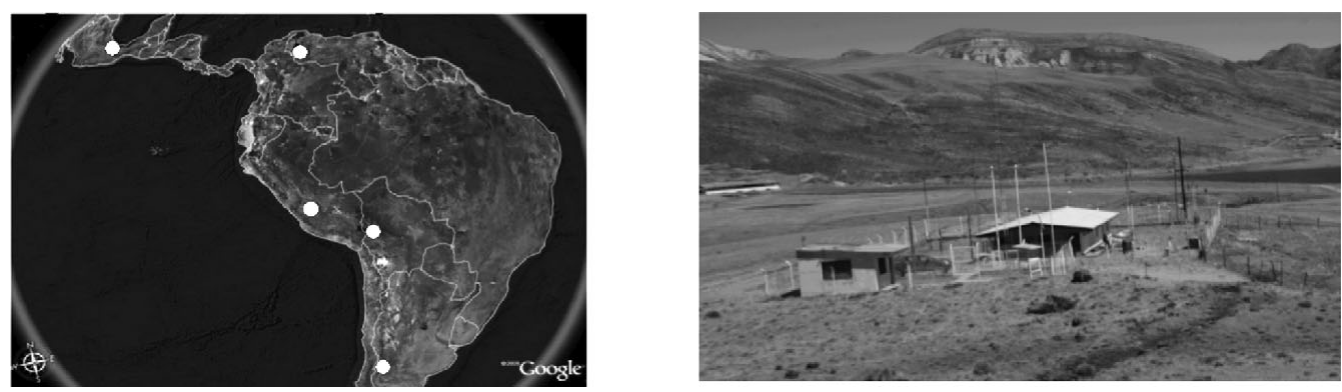

Figure 1. The left pannel hand picture shows the stations of LAGO in Sierra Negra (Mexico), Pico Espejo (Venezuela), Marcapomacocha (Peru), Chacaltaya (Bolivia) and Bariloche (Argentina). The right pannel hand picture shows a view of the Marcapomacocha site.

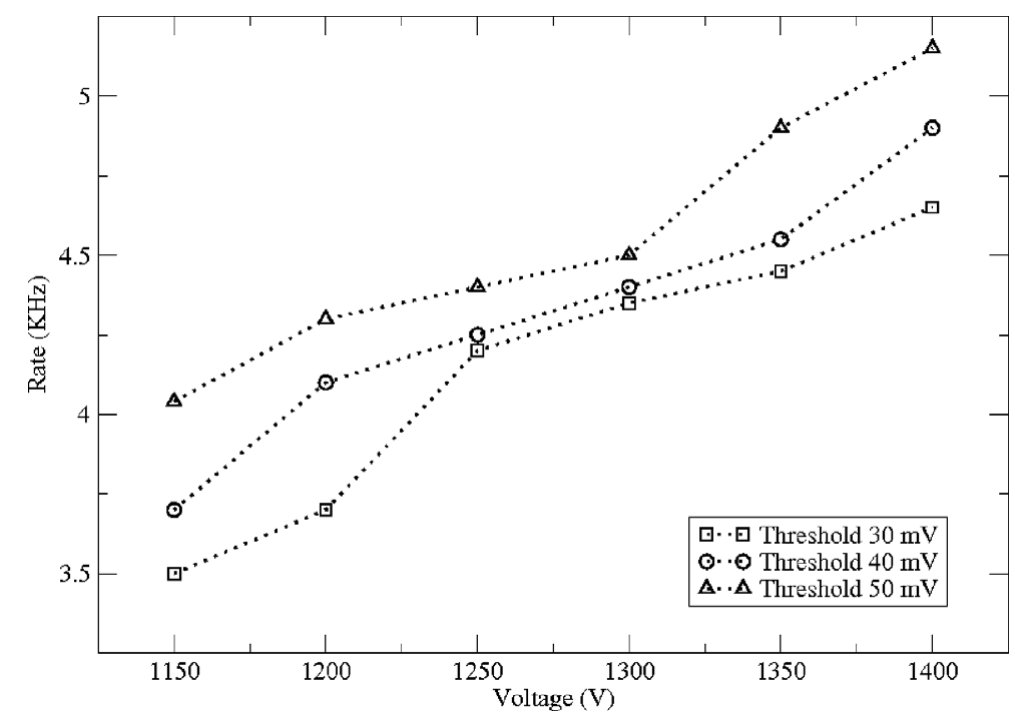

Figure 2. Total rate of charge pulses in one minute for thresholds 30,40 and $50 \mathrm{mV}$.

for about a week. After that, the water pass to the WCDs through three filters of 20, 5 and 1 micra and 2 activated carbon filters, leaving behind the sediment, finally chlorination is made inside the WCD.

The signal from the PMT is digitalized by an adquisition board (Local Station, LS) of the engineering array of the PAO. It has six channels that sampled the data at $40 \mathrm{MHz}$. The content of four scalers per channel is read out every $5 \mathrm{~ms}$. and it is send by serial line to an ARM computer.

\section{Calibration of the Detectors}

The FPGA(Field Programmable Gate Array) of the LS allows the read out of the distribution of the charge of the pulses generated in the WCD by secondary cosmic rays. We choose the high voltage applied to the PMT at $1250 \mathrm{~V}$ by the condition that the rate of cosmic rays is maintained linear in a range of $+/-50 \mathrm{~V}$ (See Fig.2). A secondary 


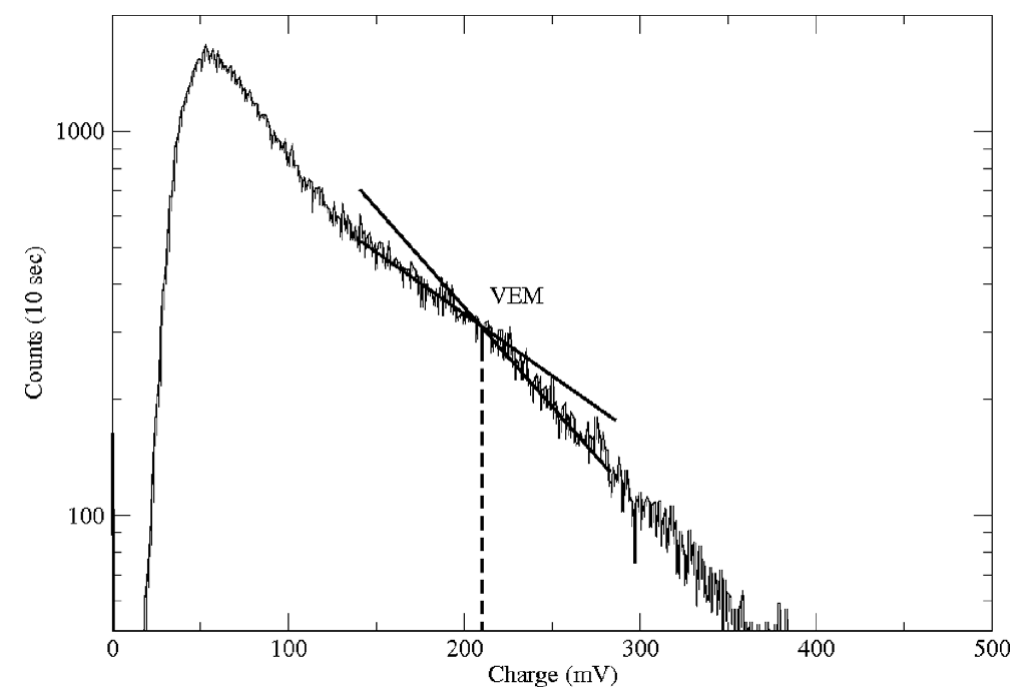

Figure 3. First calibration data: distributions of charge of the signal of the secondary cosmic rays traversing the WCD tank in 10 seconds, the VEM (Vertical Energetic Muon) position is extrapolated from the change in the slope of the charge plot).

peak in the distribution due to atmospheric vertical muons is expected at an energy of $260 \mathrm{MeV}$ (muons deposit $200 \mathrm{MeV} / \mathrm{cm}$ when they cross water). Calibration points are obtained by finding a change in the slope of the distribution of charge (see Fig. 3).

\section{Conclusions}

A first WCD of $4 \mathrm{~m}^{2}$ has been installed in Peru at 4450 m.a.s.l. at Marcapomacocha site. The procedure for water treatment and calibration of the detectors has been developed.

\section{Acknowledgements}

We would like to thank the LAGO colaboration, CONIDA (Comisión Nacional de Investigación y Desarrollo Aeroespacial del Perú) for their support to this work and the Pierre Auger Observatory for providing electronic equipment.

\section{References}

Aglietta, M. et al. 1996, Astrophys. J., 469, 305-310.

Bertou X. for the LAGO Collaboration 2008, NIM A, 595, 70-72.

Pierre Auger Collaboration 2004, NIM A, 523, 50-95. 